\title{
Kinetic Modulation of Kv4-Mediated A-Current by Arachidonic Acid Is Dependent on Potassium Channel Interacting Proteins
}

\author{
Mats H. Holmqvist, ${ }^{1}$ Jie Cao, ${ }^{1}$ Maria H. Knoppers, ${ }^{1}$ Mark E. Jurman, ${ }^{1}$ Peter S. Distefano, ${ }^{1}$ \\ Kenneth J. Rhodes, ${ }^{2}$ Yu Xie, ${ }^{1}$ and W. Frank An' ${ }^{1}$ \\ ${ }^{1}$ Millennium Pharmaceuticals Inc., Cambridge, Massachusetts 02139, and 2Wyeth-Ayerst Research, Princeton, New \\ Jersey 08543
}

The Kv4 subfamily of voltage-gated potassium channels is responsible for the transient A-type potassium current that operates at subthreshold membrane potentials to control membrane excitability. Arachidonic acid was shown recently to modulate both the peak amplitude and kinetics of the hippocampal A-current. However, in Xenopus oocytes, arachidonic acid only inhibited the peak amplitude of Kv4 current without modifying its kinetics. These results suggest the existence of Kv4 auxiliary subunit(s) in native cells. We report here a K-channel interacting protein (KChIP)-dependent kinetic modulation of Kv4.2 current in Chinese hamster ovary cells and Kv4.2 and Kv4.3 currents in Xenopus oocytes by arachidonic acid at physiological concentrations. This concentrationdependent effect of arachidonic acid resembled that observed in cerebellar granule neurons and was fully reversible. Other fatty acids, including a nonhydrolyzable inhibitor of both li-

The Kv4 voltage-gated potassium channels form fast-inactivating outward currents that underlie the somatodendritic A-current in neurons (Sheng et al., 1992; Maletic-Savatic et al., 1995; Serodio and Rudy, 1998) and the transient outward current $\left(I_{\text {to }}\right)$ in heart (Dixon et al., 1996; Xu et al., 1996; Barry et al., 1998; London et al., 1998). They operate at subthreshold membrane potentials to regulate membrane excitability (Baldwin et al., 1991; Serodio et al., 1994; Hoffman et al., 1997; Magee et al., 1998). In cardiac myocytes, suppression of $I_{\text {to }}$ results in prolonged action potential duration (Xu et al., 1999; Guo et al., 2000). In hippocampal neurons, dendritic A-current plays a critical role in controlling spatial progression and temporal integration of back-propagating action potentials and EPSPs or IPSPs, thus providing a rapid electrical signal for induction of associative events such as longterm potentiation (LTP) or long-term depression (LTD) (for review, see Magee et al., 1998; Hoffman and Johnston, 1999; Johnston et al., 2000).

Recent work has shown that in both Xenopus oocytes and neurons, the Kv4 A-type current is subject to direct modulation by arachidonic acid (Villarroel and Schwarz, 1996; Keros and McBain, 1997; Colbert and Pan, 1999). In oocytes, arachidonic

\footnotetext{
Received Nov. 20, 2000; revised March 21, 2001; accepted March 22, 2001.

We thank Melissa Brown for preparing cerebellar granule neurons, Dr. Kewei Wang for the Kv4.2 oocyte expression vector, Dr. Michael Jacobson for help with statistical analysis, Drs. Mark Bowlby, Qiang Lu, Irwin Levitan, James Trimmer, and Gary Yellen for critical comments on this manuscript, and Drs. Chris Williams, Kevin Willis, and James Barrett for encouragement and support.

Correspondence should be addressed to Dr. W. Frank An, Millennium Pharmaceuticals, Inc., 640 Memorial Drive, Cambridge, MA 02139. E-mail: an@mpi.com. Copyright (C) 2001 Society for Neuroscience $0270-6474 / 01 / 214154-08 \$ 15.00 / 0$
}

pooxygenase and cyclooxygenase, 5,8,11,14-eicosatetraynoic acid (ETYA), also mimicked arachidonic acid in modulating Kv4.3 and Kv4.3/KChIP1 currents. Compared with another transient potassium current formed by $\mathrm{Kv1.1/Kv \beta 1,Kv4.3/}$ KChIP1 current was much more sensitive to arachidonic acid. Association between KChIP1 and Kv4.2 or Kv4.3 was not altered in the presence of $10 \mu \mathrm{M}$ ETYA as measured by immunoprecipitation and association-dependent growth in yeast. Our data suggest that the KChIP proteins represent a molecular entity for the observed difference between arachidonic acid effects on A-current kinetics in heterologous cells and in native cells and are consistent with the notion that KChIP proteins modulate the subthreshold A-current in neurons.

Key words: arachidonic acid; Kv4; KChIP; fatty acids; inactivation; potassium channel; auxiliary subunit

acid inhibits Kv4 peak current amplitudes selectively compared with currents formed by $\alpha$ subunits of other $\mathrm{Kv}$ subfamilies (Honore et al., 1992; Gubitosi-Klug et al., 1995; Villarroel and Schwarz, 1996). Application of arachidonic acid to hippocampal pyramidal neurons also suppresses the A-current and enhances dendritic action potentials (Colbert and Pan, 1999) and somatic action potentials in a high- $\left[\mathrm{K}^{+}\right]_{\mathrm{o}}$ cellular model of epilepsy (Keros and McBain, 1997). In neurons, the inhibition of peak amplitude by arachidonic acid is accompanied by substantially increased rate of inactivation. However, in oocytes, the inhibition of peak amplitude by arachidonic acid isn't accompanied by kinetic changes. These observations suggest that oocytes lack a neuronal auxiliary subunit(s) that contributes to the full spectrum of pharmacological modulation of Kv4-mediated A-current by arachidonic acid.

We recently cloned K-channel interacting proteins (KChIPs) that specifically bind to the N-terminal intracellular domain of Kv4 and modulate Kv4 channel activity (An et al., 2000). The $\mathrm{KChIP}$ proteins, KChIP1, KChIP2, and KChIP3, are a group of EF-hand calcium binding proteins that belong to the neuronal calcium sensor-recoverin family. The modulatory effects of these KChIPs include enhancement of Kv4 current density, decreased rate of inactivation, and acceleration of recovery from steady state inactivation. KChIP1 and KChIP3 are brain-predominant, whereas KChIP2 is mainly expressed in both heart and brain. In this study, we tested the hypothesis that KChIPs account for the observed difference in arachidonic acid modulation of native and heterologously expressed Kv4 A-currents. We report that kinetic modulation of Kv4 by arachidonic acid, as well as by certain fatty 
acids, is indeed dependent on the presence of KChIPs. Furthermore, the Kv4.3/KChIP1 current is more sensitive than the $\mathrm{Kv} 1.1 / \mathrm{Kv} \beta 1$ current to arachidonic acid modulation. Our data provide pharmacological evidence that is consistent with KChIPs playing a critical role in the modulation of subthreshold somatodendritic A-current and dendritic excitability.

\section{MATERIALS AND METHODS}

Cell culture. Chinese hamster ovary $(\mathrm{CHO})$ cells were transfected transiently with rat Kv4.2- and/or human KChIP1-expressing plasmids using FuGENE 6 (Roche Molecular Biochemicals, Indianapolis, IN). Cerebellar granule neurons were prepared from 6-7 d postnatal Sprague Dawley rats and cultured as in Miller and Johnson (1996) for $2 \mathrm{~d}$ before being switched to Neurobasal-B27 medium (Brewer et al., 1993) supplemented with $0.5 \%$ dialyzed, heat-inactivated FBS, $50 \mu \mathrm{g} / \mathrm{ml}$ gentamicin, $0.5 \mathrm{~mm}$ L-glutamine, $20 \mathrm{~mm} \mathrm{KCl}$, and $3.3 \mu \mathrm{g} / \mathrm{ml}$ aphidicolin.

Patch-clamp recording. Standard whole-cell patch-clamp recordings were performed 1-3 d after transfection and within $7 \mathrm{~d}$ of neuronal culture. Electrodes were pulled from filamented borosilicate glass (Sutter Instruments, Novato, CA) and had an initial resistance of 3-5 M $\Omega$. The recording solution was a modified HBSS (Life Technologies, Rockville, MD) containing (in mM): $138 \mathrm{NaCl}, 0.3 \mathrm{Na}_{2} \mathrm{HPO}_{4}, 5.4 \mathrm{KCl}, 0.4 \mathrm{KH}_{2} \mathrm{PO}_{4}$, $0.9 \mathrm{MgCl}_{2}, 0.4 \mathrm{MgSO}_{4}, 1.3 \mathrm{CaCl}_{2}, 5.5 \mathrm{D}$-glucose, and $10 \mathrm{HEPES}, \mathrm{pH}$ 7.4. The intracellular electrode solution contained (in $\mathrm{mM}$ ): $140 \mathrm{KCl}, 10$ HEPES, 10 EGTA, and $0.5 \mathrm{MgCl}_{2}, \mathrm{pH} \mathrm{7.3.} \mathrm{Currents} \mathrm{were} \mathrm{evoked} \mathrm{by} \mathrm{a}$ voltage step to $+40 \mathrm{mV}$ from a holding potential of $-80 \mathrm{mV}$ and recorded using an EPC9 patch-clamp amplifier (Heka Elektronik, Lambrecht/Pfalz, Germany). All experiments were performed at room temperature.

Xenopus oocytes recording. Xenopus laevis were handled in compliance with the United States Public Health Service Policy on Humane Care and Use of Laboratory Animals and National Institutes of Health Guide for the Care and Use of Laboratory Animals, as well as with institutional animal care and use committee guidelines. Oocytes were surgically removed from the frogs under cold anesthesia and treated with type II collagenase (2-4 mg/ml) (Worthington Biochemical Corp., Lakewood, NJ) dissolved in $\mathrm{Ca}^{2+}$-free OR2 medium (in mM, $82.5 \mathrm{NaCl}, 2.5 \mathrm{KCl}, 1 \mathrm{MgCl}_{2}$, and 5 HEPES, pH 7.6) for 2 hr. cRNAs encoding rat Kv4.3, KChIP1, Kv1.1, and $\operatorname{Kv} \beta 1$ proteins were synthesized by standard in vitro transcription (Promega, Madison, WI). After a total of $50 \mathrm{nl}$ per oocyte of cRNA (1-10 ng) was microinjected, the oocytes were incubated in ND96 medium [(in mM) $96 \mathrm{NaCl}, 2 \mathrm{KCl}, 1.8 \mathrm{CaCl}_{2}, 1 \mathrm{MgCl}_{2}$, and 5 HEPES, $\mathrm{pH}$ 7.6] containing $50 \mu \mathrm{g} / \mathrm{ml}$ gentamicin at $18^{\circ} \mathrm{C}$ for $1-3 \mathrm{~d}$ before recordings. Electrodes were filled with $3 \mathrm{M} \mathrm{KCl}$ and had electrode resistances ranging from $0.2-1 \mathrm{M} \Omega$. The two-electrode voltage-clamp recordings were performed in low calcium ND96 $\left(0.3 \mathrm{mM} \mathrm{CaCl}_{2}\right)$ using a Turbo Tec 03 Clamp Amplifier (ALA Scientific Instruments, Westbury, NY). Currents were evoked with a voltage step to $+40 \mathrm{mV}$ from a holding potential of $-80 \mathrm{mV}$. The current signals were filtered at $1000 \mathrm{~Hz}$ before acquisition using the Pulse software (Heka).

Perfusion of fatty acids. Fatty acids were obtained from Sigma (St. Louis, MO) and stored as $100 \mathrm{~mm}$ stock solutions in ethanol at $-80^{\circ} \mathrm{C}$. Fresh aliquots of fatty acids were diluted into recording solution shortly before use. The vehicle $(0.01 \%$ ethanol) did not cause any changes in the peak amplitude or kinetics of Kv4 and Kv4/KChIP currents. Recordings were performed generally within the first $3 \mathrm{~min}$ of perfusion unless otherwise noted. Washout procedures were performed with ND96 supplemented with $0.5 \mathrm{mg} / \mathrm{ml} \mathrm{BSA} \mathrm{to} \mathrm{aid} \mathrm{fast} \mathrm{removal.}$

Yeast 2-hybrid strains and growth assays. Diploid strains containing bait (the N-terminal domain of Kv4.3 or the empty vector pGBT9) and fish (KChIP1) plasmids were obtained as described in An et al. (2000). For synchronization, strains were grown to saturation before they were inoculated at equal cell densities as measured by OD600 values. Cells were placed into $5 \mathrm{ml}$ of synthetic complete-TrpLeuHis drop-out (SC$\mathrm{WLH}$ ) medium that selects for interaction-dependent growth or $5 \mathrm{ml}$ of SC-WL medium that is nonselective in the presence or absence of $10 \mu \mathrm{M}$ 5,8,11,14-eicosatetraynoic acid (ETYA). Five millimolar 3-amino-1,2,4triazole was included in the media to suppress weak self-activating activity from the Kv4.3 N-terminal domain bait. Cultures were grown for $17 \mathrm{hr}$ at $30^{\circ} \mathrm{C}$, and OD600 values were read by a spectrophotometer.

Immunoprecipitation and immunoblotting. Human embryonic kidney (HEK) 293T cells were transfected with Kv4.2- and KChIP1-expressing plasmids using Polyfect (Qiagen, Valencia, CA). Cells were lysed $48 \mathrm{hr}$ after transfection in lysis buffer containing $0.5 \% \mathrm{NP}-40,120 \mathrm{~mm} \mathrm{NaCl}, 1$ mm EDTA, and $50 \mathrm{~mm}$ TrisCl, $\mathrm{pH}$ 8.0. Cell lysate was divided equally and treated with or without $10 \mu \mathrm{M}$ ETYA in the immunoprecipitation reactions using anti-Kv4.2 antibody as described in An et al. (2000). Immunoprecipitation products were separated on 4-20\% SDS-PAGE and immunoblotted with anti-KChIP1 antibody as described in An et al. (2000).

Data and statistical analysis. All inactivation time constants were obtained by fitting a single exponential function to the decaying phase of currents. Differences between treatment groups were established by ANOVA followed by Bonferroni correction for multiple post hoc comparisons. Results were deemed statistically significant at $p<0.05$.

\section{RESULTS}

\section{KChIP-dependent modulation by arachidonic acid in heterologous cells mimics arachidonic acid effects in cerebellar granule neurons}

We first investigated arachidonic acid effects on the A-current in a neuronal system (cultured primary cerebellar granule neurons) in which both Kv4 and KChIPs are present. Considerable evidence suggests that the A-current in these neurons is predominantly formed by Kv4-family $\alpha$ subunits (Sheng et al., 1992; Serodio and Rudy, 1998; An et al., 2000). TEA (10 mm) was applied to block a small sustained outward component. As shown in Figure $1, A$ and $E$, the inactivation time constant of the A-current was reduced by $52 \%$ (from $44 \pm 5$ to $21 \pm 3 \mathrm{msec}$ ) after application of $10 \mu \mathrm{M}$ arachidonic acid. The corresponding peak amplitude was reduced from $2.0 \pm 0.6$ to $1.2 \pm 0.4 \mathrm{nA}$ (Fig. 1D). These results confirm that arachidonic acid modulates both Kv4 A-current kinetics and amplitude in native cells. Although the inactivation time constant of A-current in cerebellar granule neurons reported here is higher than that in hippocampal neurons [for example, $\sim 23 \mathrm{msec}$ as reported in Keros and McBain (1997)], arachidonic acid at $10 \mu \mathrm{M}$ reduced the inactivation time constants to approximately one half of its initial values in both cases when currents were evoked by depolarizations to $+40 \mathrm{mV}$.

Next, we tested Kv4.2 current expressed ectopically in a nonneuronal mammalian system ( $\mathrm{CHO}$ cells). When Kv4.2 was expressed alone, the peak amplitude of the resulting current was reduced by $40 \%$ by $10 \mu \mathrm{M}$ arachidonic acid compared with control (from $0.62 \pm 0.08$ to $0.37 \pm 0.11 \mathrm{nA}$ ) (Fig. $1 B, D$ ). However, the inactivation time constant remained unchanged (Fig. 1B,E). These data are consistent with previous observations in Xenopus oocytes that arachidonic acid effects on currents formed by Kv4 subfamily $\alpha$ subunit alone were restricted to modulation of peak amplitude (Villarroel and Schwarz, 1996).

To test whether the KChIP proteins represent the molecular basis for kinetic modulation of the Kv4 A-current by arachidonic acid in neurons, we coexpressed KChIP1 with Kv4.2 in CHO cells. In contrast to the current formed by Kv4.2 expressed alone, current formed by Kv4.2 and KChIP1 demonstrated a marked kinetic sensitivity to arachidonic acid. In the absence of arachidonic acid, the inactivation time constant of Kv4.2/KChIP1 current was $88 \pm 8 \mathrm{msec}$, consistent with our previous observations (An et al., 2000). However, after application of $10 \mu \mathrm{M}$ arachidonic acid, the inactivation time constant was reduced by $58 \%$ to $37 \pm$ $3 \mathrm{msec}$ (Fig. 1C,E). The peak amplitude of Kv4.2/KChIP1 current was also inhibited by $38 \%$ (from $4.5 \pm 0.4$ to $2.8 \pm 0.5 \mathrm{nA}$ ), a magnitude consistent with the inhibition of Kv4.2 alone by arachidonic acid. Similar results were obtained when Kv4.2 was coexpressed with KChIP1 in Xenopus oocytes (Fig. 4) or when $\mathrm{Kv} 4.3$ was coexpressed with KChIP2 in oocytes (data not shown). Therefore, we conclude that modulation of $\mathrm{Kv} 4$ inactivation by arachidonic acid is KChIP-dependent, but that the arachidonic acid effect on Kv4 current amplitude is independent of KChIPs. 
A

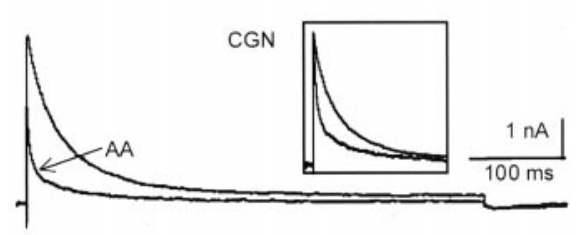

B

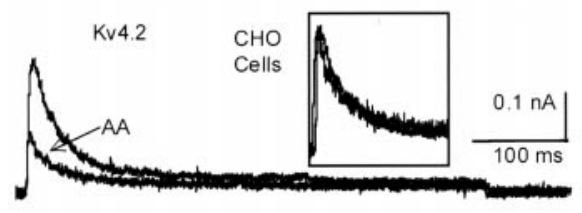

C
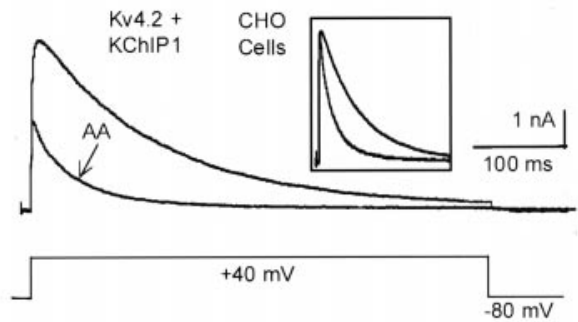

D

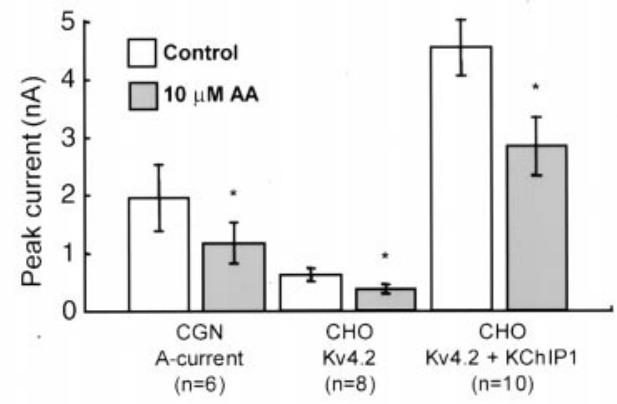

$E$

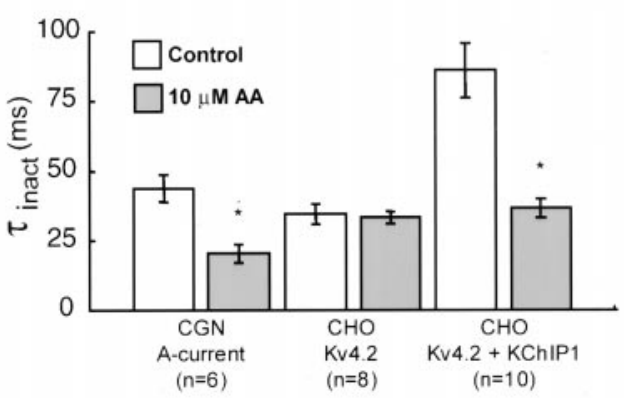

Figure 1. KChIP-dependent modulation of Kv4.2 by arachidonic acid in heterologous cells mimics that of cerebellar A-current. $A-C$, Outward potassium currents were recorded by whole-cell patch clamp in cultured primary cerebellar granule neurons $(C G N)$ in the presence of $10 \mathrm{~mm}$ TEA $(A)$, and in $\mathrm{CHO}$ cells transfected with $\mathrm{Kv} 4.2$ alone $(B)$ or cotransfected with Kv4.2 and KChIP1 $(C)$. Insets show normalized, scaled recording traces for comparison. Holding potentials were $-80 \mathrm{mV}$, and cells were depolarized with a single pulse to $+40 \mathrm{mV}$ (duration, 500 $\mathrm{msec}$ ). Recordings in the presence of $10 \mu \mathrm{M}$ arachidonic acid are indicated by $A A$. $D, E$, Modulations of peak current amplitudes $(D)$ and inactivation time constants $\left(\tau_{\text {inact }}\right)(E)$ by $10 \mu \mathrm{M}$ arachidonic acid (shaded bars) for cells in $A-C$. Values are presented as mean \pm SEM. * indicates statistically significant differences between controls and arachidonic acid-treated cells, and $n$ indicates the number of cells recorded.

\section{Arachidonic acid modulation of Kv4/KChIP current is concentration-dependent and reversible}

We studied the effects of different concentrations of arachidonic acid on Kv4.3/KChIP1 current in Xenopus oocytes. Because the
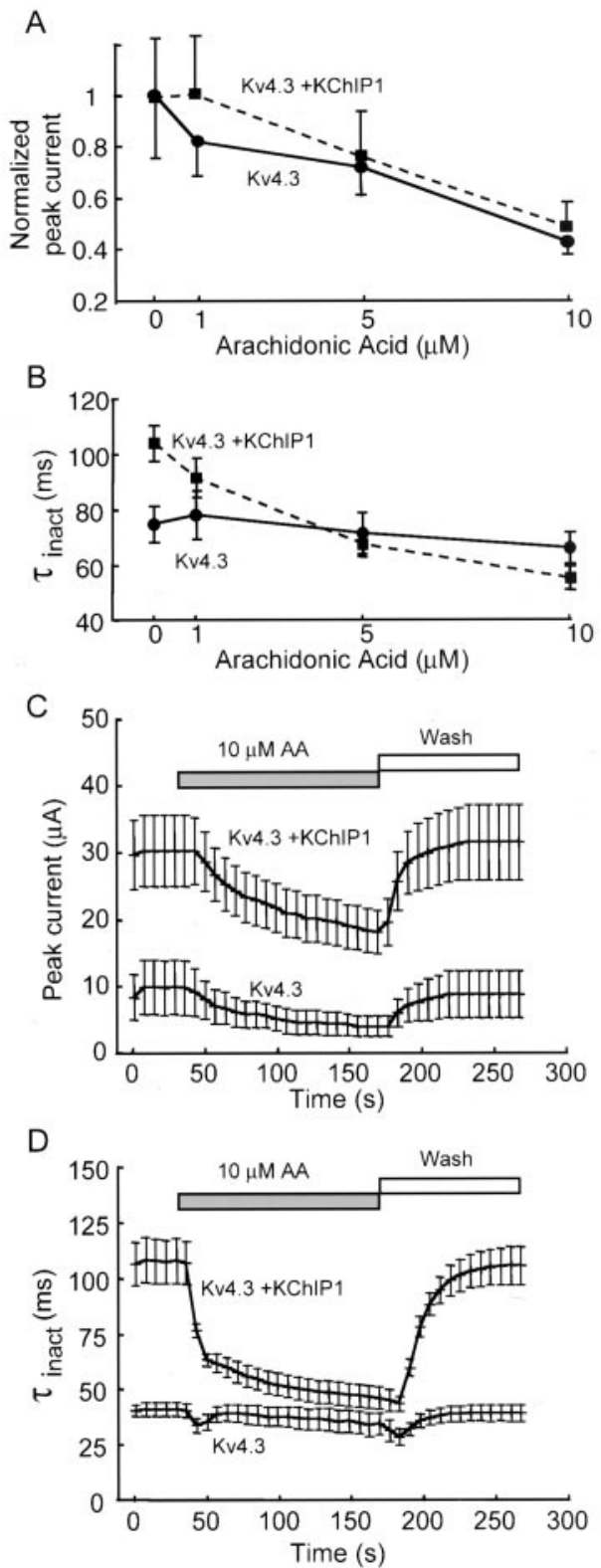

Figure 2. Concentration-dependent and reversible modulation of Kv4.3 and Kv4.3/KChIP1 currents in Xenopus oocytes by arachidonic acid. Depolarizing pulses from a holding potential of -80 to $+40 \mathrm{mV}$ (duration, $500 \mathrm{msec}$ ). Arachidonic acid at 1-10 $\mu \mathrm{M}$ inhibited peak current amplitudes $(A$, normalized to values at $0 \mu \mathrm{M}$ arachidonic acid) in oocytes injected with Kv4.3 cRNA alone (solid line) and coinjected with both Kv4.3 and KChIP1 cRNA (dashed line), but only decreased inactivation time constants $\left(\tau_{\text {inact }}\right)$ in $\mathrm{Kv} 4.3 / \mathrm{KChIP} 1$ coinjected oocytes $(B) . C, D$, Time course of arachidonic acid modulation of Kv4.3 and Kv4.3/KChIP1. Currents in Xenopus oocytes were evoked every $7 \mathrm{sec}$ with depolarizing pulses to $+40 \mathrm{mV}$ (duration, $500 \mathrm{msec}$ ) from a holding potential of -80 $\mathrm{mV}$. Effects on peak current amplitude $(C)$ and inactivation time constants $\left(\tau_{\text {inact }}\right)(D)$ are shown with shaded bars indicating application of 10 $\mu \mathrm{M}$ arachidonic acid and open bars indicating washout with ND96 medium supplemented with $0.5 \mathrm{mg} / \mathrm{ml}$ BSA. $n=5$ oocytes for each data point.

physiological concentrations of arachidonic acid are often $<10 \mu \mathrm{M}$ (Needleman et al., 1986; Anderson and Welsh, 1990; Meves, 1994), we chose to test arachidonic acid in the $1-10 \mu \mathrm{M}$ range. The concentration-dependent block of peak amplitude of the Kv4.3 current was independent of the presence of KChIP1 (Fig. 2A). Furthermore, the slope of amplitude reduction as a function of 
increasing concentrations was very similar with or without KChIP1. Peak current block did not appear to saturate up to 10 $\mu \mathrm{M}$. Villarroel and Schwarz (1996) reported that the $\mathrm{IC}_{50}$ of arachidonic acid on Kv4 $\alpha$ subunits was $\sim 8 \mu \mathrm{M}$ in oocytes. The inactivation time constant in the absence of KChIP1 was unchanged at all arachidonic acid concentrations tested. However, in the presence of $\mathrm{KChIP1}$, inactivation time constant decreased in a concentration-dependent manner (Fig. 2B).

The kinetics of the two effects of arachidonic acid on total current and on inactivation were quite different. The amplitude block developed gradually over time (Fig. 2C). The presence of KChIP1 did not substantially alter either the percentage decrease or the rate of current block over time, nor did it change the rate of recovery of Kv4.3 current amplitude over time (Fig. $2 C$ ). In contrast to the gradual development of amplitude block, the KChIP1-dependent effect on Kv4 inactivation appeared much more rapidly after arachidonic acid perfusion and tended to plateau quickly (Fig. 2D). When arachidonic acid was washed out, Kv4.3 current amplitude and inactivation time constant recovered fully with similar rates in the presence of KChIP1 (Fig. 2, compare $C, D)$. The two small inflections in the Kv4.3 alone plot in panel $D$ were artifacts attributable to buffer changes.

\section{Modulation of Kv4/KChIP current by other fatty acids}

Certain fatty acids were shown previously to mimic the effects of arachidonic acid on Kv4 currents in Xenopus oocytes when Kv4 $\alpha$ subunits were expressed alone (Villarroel and Schwarz, 1996). Thus, we investigated the fatty acid selectivity for Kv4 current in the presence of KChIPs. Arachidonic acid is a 20-carbon fatty acid carrying four cis double bonds with the first double bond at C5 (20:4 c5). We chose to test the following arachidonic acid analogs with distinct structural features: $\gamma$-linolenic acid (18:3 c9) has three $c i s$ double bonds instead of four double bonds, linolelaidic acid (18:2 t9) has two trans double bonds instead of four cis double bonds, ETYA (20:4 n5) has four triple bonds instead of double bonds found in arachidonic acid ( $\mathrm{n}$ indicates position of the first triple bond), and 5,8,11-eicosatriynoic acid (ETI, 20:3 n5) has three triple bonds. Figure $3 A$ shows that the peak amplitude of Kv4.3 current was inhibited significantly compared with nofatty acid control by $10 \mu \mathrm{M}$ of $\gamma$-linolenic acid, ETI, ETYA, and arachidonic acid, independent of the presence of KChIP1. The percentage inhibition of amplitude of Kv4.3 alone and Kv4.3/ KChIP1 was not significantly different for these fatty acids. A small, statistically significant block of Kv4.3 current amplitude by $10 \mu \mathrm{M}$ linolelaidic acid was observed in the presence of KChIP1 (but not in the absence) when the values were compared with their respective controls; however, there was no significant difference when comparing Kv4.3 and Kv4.3/KChIP1. The effects of these fatty acids on amplitude of Kv4 current expressed in the absence of KChIPs was consistent with what was reported previously (Villarroel and Schwarz, 1996). In the absence of KChIP1, none of the fatty acids tested showed a statistically significant effect on Kv4.3 inactivation time constant (Fig. 3B). Only those fatty acids that caused a substantial current block independently of KChIPs ( $\gamma$-linolenic acid, ETI, ETYA, and arachidonic acid) reduced the Kv4.3 inactivation time constant when coexpressed with KChIP1. Linolelaidic acid, which showed only a modest KChIP-dependent Kv4.3 current block, did not affect the Kv4.3 inactivation time constant significantly (Fig. $3 B$ ). Therefore, certain long-chain fatty acids can imitate the modulatory effects of arachidonic acid on Kv4 current kinetics in a KChIP-dependent manner. In general, there is good correlation between the ability
A

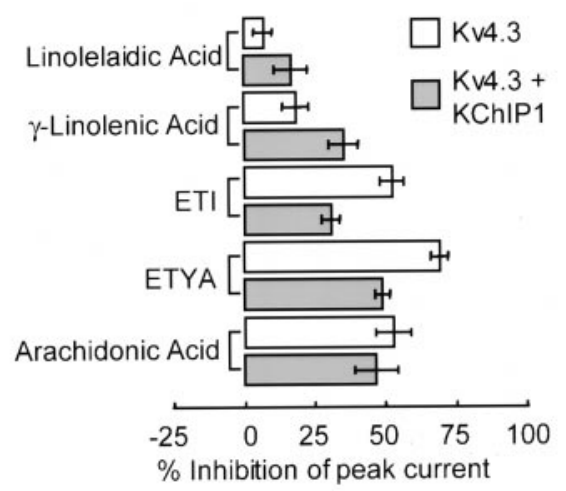

B

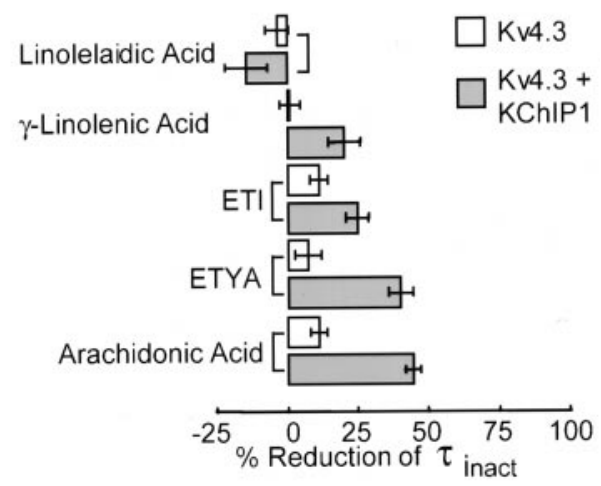

Figure 3. Modulation of $\mathrm{Kv} 4.3$ and $\mathrm{Kv} 4.3 / \mathrm{KChIP} 1$ currents by fatty acids. A, Percentage block of Kv4.3 (open bars) and Kv4.3/KChIP (shaded bars) peak current amplitudes by $10 \mu \mathrm{M}$ linolelaidic acid, $\gamma$-linolenic acid, ETI, ETYA, and arachidonic acid in Xenopus oocytes. All values except that of linolelaidic acid/Kv4.3 alone were statistically significant when compared with no fatty acid controls. Comparisons between $\mathrm{Kv} 4.3$ and $\mathrm{Kv} 4.3 / \mathrm{KChIP} 1$ for all fatty acids were not statistically significant. $B$, Percentage inhibition of inactivation time constants $\left(\tau_{\text {inact }}\right)$ of currents in $A$ under the same treatments. All values for $\mathrm{Kv} 4.3$ alone were not significant compared with no fatty acid control. All values for Kv4.3/ KChIP1 except that of linolelaidic acid were statistically significant compared with no fatty acid control. The differences of values between Kv4.3 and $\mathrm{Kv} 4.3 / \mathrm{KChIP} 1$ for every fatty acid treatment except linolelaidic acid were significant. Values are presented as mean \pm SEM; $n=4-9$ oocytes per treatment.

of a given fatty acid to block peak amplitude and to modify kinetics of the reconstituted $\mathrm{Kv} 4 / \mathrm{KChIP}$ current.

\section{$\mathrm{Kv} 4 / \mathrm{KChIP}$ current is more sensitive than $\mathrm{Kv} 1.1 / \mathrm{Kv} \beta 1$ current to arachidonic acid modulation}

We next wanted to determine whether arachidonic acid would modulate preferentially the Kv4 subfamily as opposed to other $\mathrm{Kv}$ subfamilies. Previous work in Xenopus oocytes compared the effects of arachidonic acid on currents formed by the $\alpha$ subunits alone of $\mathrm{Kv} 1, \mathrm{Kv} 2, \mathrm{Kv} 3$, and $\mathrm{Kv} 4$, and found that $\mathrm{Kv} 4$ currents were by far the most sensitive to arachidonic acid (Villarroel and Schwarz, 1996). However, in the case of Kv1 subfamily channels, multiple $\beta$ subunits $(\mathrm{Kv} \beta \mathrm{s})$ exist that can dramatically change current density and kinetic properties of Kv1 $\alpha$ subunits (Rettig et al., 1994; Scott et al., 1994; Shi et al., 1996; Pongs et al., 1999). Although Villarroel and Schwarz (1996) reported only modest effects of arachidonic acid on Kv1 subfamily channels, an obser- 
A

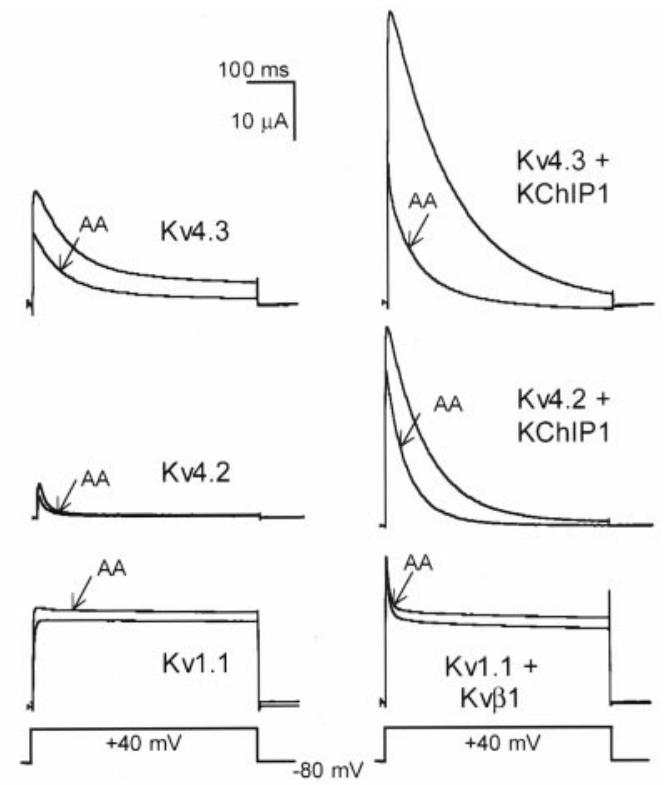

B
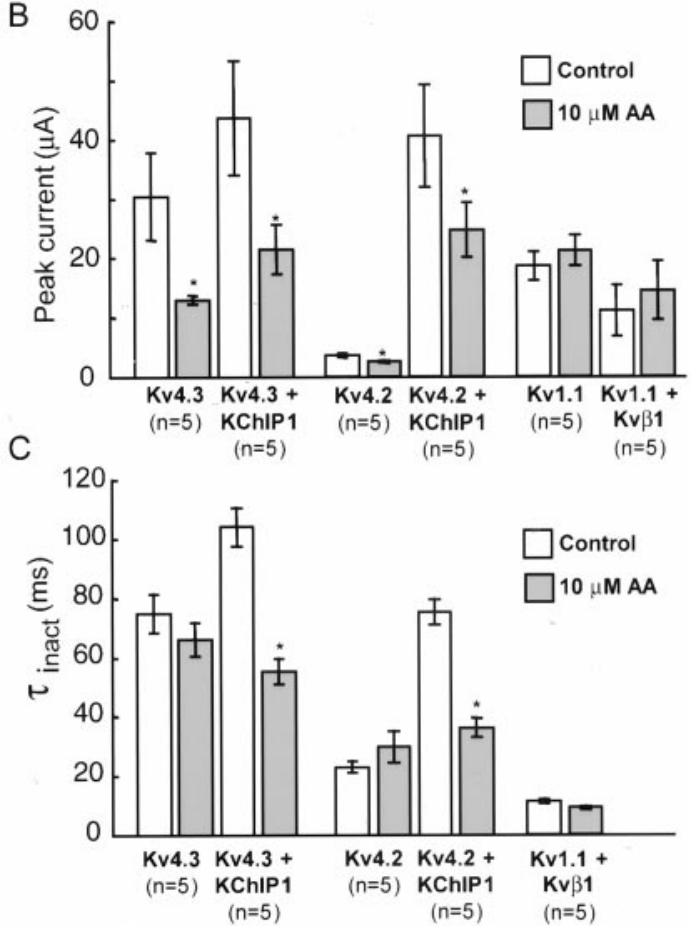

Figure 4. $\mathrm{Kv} 4 / \mathrm{KChIP}$ current is more sensitive than $\mathrm{Kv} 1.1 / \mathrm{Kv} \beta 1$ to arachidonic acid modulation. Representative recording traces $(A)$ from Xenopus oocytes injected with cRNAs for Kv4.2 alone, Kv4.2/KChIP1, $\mathrm{Kv} 4.3$ alone, $\mathrm{Kv} 4.3 / \mathrm{KChIP} 1, \mathrm{Kv} 1.1$ alone, and $\mathrm{Kv} 1.1 / \mathrm{Kv} \beta 1$ in the presence (arrows) and absence of arachidonic acid $(A A)$. Summary of arachidonic acid effects on peak current amplitudes $(B)$ and inactivation time constants $\left(\tau_{\text {inact }}\right)(C)$ of currents in $A$. Values are mean \pm SEM; $n$ indicates the number of oocytes recorded.

vation similar to that described by Gubitosi-Klug et al. (1995) of arachidonic acid effects on Kv1.1 current in insect sf9 cells, these reports left unanswered the question whether the presence of $\mathrm{Kv} \beta$ s would change the amplitude and kinetic sensitivity of Kv1 channels to arachidonic acid. Because the Kv1 subfamily is the only other group of mammalian $\mathrm{Kv}$ channels for which auxiliary subunits have so far been identified, we tested and compared sensitivities of reconstituted Kv4.3/KChIP1 and $\mathrm{Kv} 1.1 / \mathrm{Kv} \beta 1$ channels to arachidonic acid in Xenopus oocytes.

As shown in Figure 4, the presence of $\operatorname{Kv} \beta 1$ did not result in significant changes in the way the Kv1.1 $\alpha$ subunit responded to arachidonic acid. Kv1.1 current amplitudes were $19 \pm 2 \mu \mathrm{A}$ before and $21 \pm 3 \mu \mathrm{A}$ after application of $10 \mu \mathrm{M}$ arachidonic acid, and those of $\mathrm{Kv} 1.1 / \mathrm{Kv} \beta 1$ were $11 \pm 4 \mu \mathrm{A}$ before and $14 \pm 1 \mu \mathrm{A}$ after application of $10 \mu \mathrm{M}$ arachidonic acid (Fig. 4A,B). The inactivation time constant of the transient component of Kv1.1 current, measured in the presence of $\mathrm{Kv} \beta 1$, was not significantly decreased after the application of arachidonic acid (Fig. 4C). We could not measure the inactivation time constants in the absence of $\operatorname{Kv} \beta 1$ because of the noninactivating nature of the current, and we did not observe qualitative changes in current kinetics (Fig. $4 A$ ). In contrast to $\mathrm{Kv} 1.1 / \mathrm{Kv} \beta 1,10 \mu \mathrm{M}$ arachidonic acid produced a very robust modulation of $\mathrm{Kv} 4.3 / \mathrm{KChIP} 1$ currents with $52 \%$ block of peak amplitude (from $44 \pm 10$ to $21 \pm 4 \mu \mathrm{A}$ ) (Fig. $4 B$ ) and $47 \%$ reduction of inactivation time constant (from $104 \pm 7$ to $55 \pm 4 \mathrm{msec}$ ) (Fig. 4C). Kv4.3 current amplitude was also blocked by $57 \%(30 \pm 7 \mu \mathrm{A}$ before vs $13 \pm 1 \mu \mathrm{A}$ after application of arachidonic acid) without accompanied changes in the rate of inactivation in the absence of KChIP1. Similar results were obtained with Kv4.2 (Fig. 4). We conclude that Kv4.3 and Kv4.2 currents are much more sensitive to arachidonic acid than those of Kv1.1 either alone or in combination with their cognate auxiliary subunits.

\section{ETYA does not disrupt association of KChIP1 and Kv4}

KChIPs have been shown to associate with the N-terminal intracellular domains of $\mathrm{Kv} 4 \alpha$ subunits, increase $\mathrm{Kv} 4$ current density, and slow inactivation (An et al., 2000). In the present study, we have shown that arachidonic acid reduces the current amplitude of $\mathrm{Kv} 4$ and accelerates the rate of inactivation, an apparent reversal of the KChIP effects. This raises the possibility that arachidonic acid acts by interfering with the binding between Kv4 and KChIPs. To test this hypothesis, we first used the yeast two-hybrid system to test whether ETYA would alter the physical interaction between $\mathrm{KChIP} 1$ and $\mathrm{Kv} 4.3 \mathrm{~N}$-terminal domain (Kv4.3N). Yeast strains coexpressing KChIP1 (fish) and either the N-terminal domain of Kv4.3 (bait) or the empty bait vector (pGBT9) were grown in the presence or absence of $10 \mu \mathrm{M}$ ETYA. As shown in Figure 5A, KChIP1-Kv4.3N interaction-dependent growth in the selective SC-WLH medium was not affected by 10 $\mu \mathrm{M}$ ETYA. We then determined whether ETYA disrupted the association of KChIP1 and the full-length Kv4 channel. To test this, HEK 293T cells were cotransfected with Kv4.2- and KChIP1-expressing plasmids, and cell lysate was immunoprecipitated with anti-Kv4.2 antibody in the presence or absence of 10 $\mu \mathrm{M}$ ETYA. As indicated by KChIP1 immunoblot analysis, $10 \mu \mathrm{M}$ ETYA did not alter the association between the Kv4.2 channel and KChIP1 expressed in HEK293T cells (Fig. 5B). We used ETYA instead of arachidonic acid because, although both affect Kv4 current nearly identically, ETYA is nonmetabolizable, and thus is better suited for these experiments. Taken together, the results show that ETYA does not disrupt association between KChIP1 and Kv4 channels.

\section{DISCUSSION}

In this report we describe the modulation by arachidonic acid, as well as other fatty acids, of currents formed by Kv $4 \alpha$ and their KChIP auxiliary subunits. Application of physiologically relevant concentrations of arachidonic acid increased considerably the rate 
A

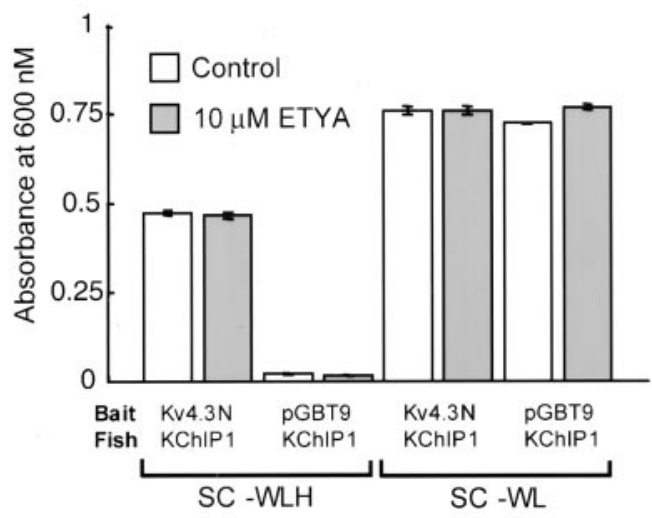

B

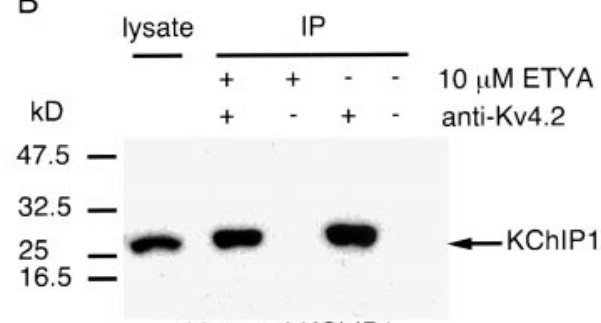

blot: anti-KChIP1

Figure 5. ETYA does not interfere with association between KChIP1 and Kv4. A, KChIP1 and N-terminal domain of Kv4.3 interactiondependent growth in selective SC-WLH medium was not altered by 10 $\mu \mathrm{M}$ ETYA. The nonselective medium SC-WL, which allowed strains to grow independently of the interaction between the N-terminal domain of Kv4.3 and KChIP1, was used to control the nonspecific effects of ETYA on growth of the strains. pGBT9 is the bait expression vector. Values are presented as mean $\pm \mathrm{SEM} ; n=4$ for each data point. $B$, The association between KChIP1 and the Kv4.2 channel remained unchanged in the presence of $10 \mu \mathrm{M}$ ETYA as determined by immunoprecipitation. Note that the intensities of the coimmunoprecipitated KChIP1 signal were not altered by the presence $(+)$ of ETYA in the immunoprecipitation $(I P)$ reactions.

of inactivation of the A-current of cerebellar granule neurons. Likewise, arachidonic acid also altered the ectopically expressed Kv4.2 currents in $\mathrm{CHO}$ cells and Kv4.2 and Kv4.3 currents in oocytes in a KChIP-dependent manner. In addition, peak current amplitudes were inhibited by arachidonic acid, but this effect was independent of the presence of KChIP subunits. Compared with $\mathrm{Kv} 4.3 / \mathrm{KChIP} 1$ and $\mathrm{Kv} 4.2 / \mathrm{KChIP} 1$, the fast inactivating potassium channel formed by the $\alpha-\beta$ subunits of $\mathrm{Kv} 1.1 / \mathrm{Kv} \beta 1$ was not significantly modulated by arachidonic acid. Finally, ETYA, a nonhybrolyzable analog of arachidonic acid, did not appear to exert its effects on $\mathrm{Kv} 4 / \mathrm{KChIP} 1$ channels by blocking the association of KChIP1 and Kv4.

Our present data are consistent with a role of KChIPs modulating the native A-current operating at subthreshold membrane potentials. The evidence for the existence of auxiliary subunit(s) in native neurons was first provided by the observation that recombinant $\mathrm{Kv} 4$ currents expressed in heterologous cells were kinetically different from the native A-current believed to be formed by Kv4 $\alpha$ subunits (Rudy et al., 1988; Chabala et al., 1993; Serodio et al., 1994, 1996). Furthermore, the difference in the above scenarios could be corrected when small molecular weight mRNA molecules extracted from brain were coinjected with Kv4 cRNA into oocytes (Rudy et al., 1988; Chabala et al., 1993; Serodio et al., 1994, 1996). We recently identified the KChIPs and showed that they associate and colocalize with Kv4 proteins both in heterologous cells and in brain, and that the reconstituted $\mathrm{Kv} 4 / \mathrm{KChIP}$ current was similar in many respects to that observed when Kv4 was reconstituted with the small molecular weight mRNA (An et al., 2000). As shown here, our data suggest $\mathrm{KChIPs}$ are responsible for rendering Kv4 inactivation kinetics susceptible to modulation by arachidonic acid. These KChIPdependent effects reconcile the previously observed difference between modulations by arachidonic acid on recombinantly expressed and native $\mathrm{Kv} 4$ channels. Although the absolute values of the inactivation time constants of $\mathrm{Kv} 4 \mathrm{~A}$-current obtained from different systems vary, which may be attributable to variation in cell types and/or other unidentified cellular factors that modulate the Kv4 current, the magnitude of reductions of the inactivation time constants in the presence of arachidonic acid is very similar. Together, KChIPs appear to be at least one group of auxiliary proteins that modulate the subthreshold A-current in neurons, and our finding of KChIP-dependent kinetic modulation of Kv4 by arachidonic acid provides a unique example in which cytoplasmic auxiliary subunits of voltage-gated ion channels modify kinetic properties of the $\alpha$ subunits in response to physiological agents.

Arachidonic acid has been shown to modulate many ion channels (for review, see Meves, 1994). Among Kv channels, however, there appears to be some level of selectivity when tested at physiological concentrations $(<10 \mu \mathrm{M})$ (Honore et al., 1992; Gubitosi-Klug et al., 1995; Villarroel and Schwarz, 1996). Villarroel and Schwarz (1996) have shown that Kv4 currents were the most sensitive compared with subclasses of Kv1, Kv2, and Kv3 channels; however, only $\alpha$ subunits of these channels were tested for arachidonic acid effects. Our present work showed that when $\alpha$ and auxiliary subunits were coassembled, the Kv4/KChIP current responded much more robustly to arachidonic acid than the $\mathrm{Kv} 1.1 / \mathrm{Kv} \beta 1$ current both in amplitude and in kinetics. The modulations of Kv1 and Kv4 channels by arachidonic acid are likely to employ distinct mechanisms. For example, arachidonic acid caused a significant reduction of amplitude of Kv4/KChIP, but a nonsignificant increase of $\mathrm{Kv} 1.1 / \mathrm{Kv} \beta 1$ amplitude (Fig. 4A,B). Also, unlike KChIPs, the presence of $\mathrm{Kv} \beta 1$ did not seem to change the response of Kv1.1 $\alpha$ subunits to arachidonic acid. The small increases of peak amplitude of Kv1.1 and $\mathrm{Kv} 1.1 / \mathrm{Kv} \beta 1$ in this work were comparable in magnitude with those of Kv1.1 $\alpha$ subunits alone in response to arachidonic acid (Fig. 4A,B) (Gubitosi-Klug et al., 1995; Villarroel and Schwarz, 1996).

Several lines of evidence suggest that arachidonic acid acts directly on $\mathrm{Kv} 4 / \mathrm{KChIP}$ to alter current properties rather than through its metabolites. First, ETYA, a nonhydrolyzable analog of arachidonic acid and an inhibitor of both lipooxygenase and cyclooxygenase, mimicked arachidonic acid effects on $\mathrm{Kv} 4 /$ KChIP current. Second, the effects of arachidonic acid could be readily reversed by washout. This is inconsistent with effects through covalent protein modifications, such as phosphorylationdephosphorylation. Third, the kinetic effects developed rapidly (within $14 \mathrm{sec}$ ) with the onset of arachidonic acid perfusion. Allowing for transit of solution through the perfusion apparatus, the effects were almost immediate. This rapid time course is consistent with direct binding of arachidonic acid to the channel. These results, coupled with the fact that the A-current from hippocampal neurons is modulated by arachidonic acid in a cellfree patch (Keros and McBain, 1997), strongly indicate a direct action of arachidonic acid on the Kv4/KChIP channel. It is at present unclear how many binding sites for arachidonic acid exist 
or where the binding sites reside on the $\mathrm{Kv} 4 / \mathrm{KChIP}$ complex. It is clear that the amplitude block and the kinetic modulation by arachidonic acid are two separable processes based on KChIPdependence, as well as on the distinct time course of development (Fig. 2C,D). It is likely that one arachidonic acid binding site is on Kv4 $\alpha$ subunit and that KChIP-independent binding of arachidonic acid to this site blocks flow of potassium ions. Additional binding site(s) may exist on the Kv4/KChIP complex to exert the distinct kinetic effects in a KChIP-dependent manner. Alternatively, the same receptor site that blocks the flow of ions may interfere with the kinetic modulation of Kv4 by KChIPs. Our data argue against the likelihood that arachidonic acid modulates Kv4 kinetic effects by disrupting the physical association between the Kv4 and KChIPs. It would be of interest to investigate whether the existing Kv4 mutations that affect the inactivation processes (Jerng and Covarrubias, 1997; Jerng et al., 1999) would alter the arachidonic acid effects.

Various neurotransmitters cause intracellular release of arachidonic acid via activation of phospholipase A2 or combined activity of phospholipase C and diglyceride lipase (Dumuis et al., 1988, 1990; Axelrod, 1990; Bito et al., 1994). Tissue insults, such as brain or heart ischemia (Bazan, 1989; Kim and Duff, 1990; Madden et al., 1996) and those resulting from seizures (Bazan et al., 1986, 1995; Siesjo et al., 1989), are known to elevate arachidonic acid levels. Arachidonic acid has been demonstrated to play a role in LTP (Drapeau et al., 1990; Miller et al., 1992; Bazan et al., 1995). The somatodendritic A-current, which is modulated by arachidonic acid, also contributes to LTP/LDP by integrating back-propagating action potentials and synaptic potentials. We show in this paper that the full spectrum of modulation by arachidonic acid of the A-current is KChIP-dependent, suggesting that KChIPs contribute to the mechanism by which arachidonic acid impacts synaptic plasticity through a series of excitatory and inhibitory ion channels.

\section{REFERENCES}

An WF, Bowlby MR, Betty M, Cao J, Ling HP, Mendoza G, Hinson JW, Mattsson KI, Strassle BW, Trimmer JS, Rhodes KJ (2000) Modulation of A-type potassium channels by a family of calcium sensors. Nature 403:553-556.

Anderson MP, Welsh MJ (1990) Fatty acids inhibit apical membrane chloride channels in airway epithelia. Proc Natl Acad Sci USA 87:7334-7338.

Axelrod J (1990) Receptor-mediated activation of phospholipase A2 and arachidonic acid release in signal transduction. Biochem Soc Trans 18:503-507.

Baldwin TJ, Tsaur ML, Lopez GA, Jan YN, Jan LY (1991) Characterization of a mammalian cDNA for an inactivating voltage-sensitive $\mathrm{K}+$ channel. Neuron 7:471-483.

Barry DM, Xu H, Schuessler RB, Nerbonne JM (1998) Functional knockout of the transient outward current, long-QT syndrome, and cardiac remodeling in mice expressing a dominant-negative Kv4 alpha subunit. Circ Res 83:560-567.

Bazan NG (1989) Arachidonic acid in the modulation of excitable membrane function and at the onset of brain damage. Ann NY Acad Sci 559:1-16.

Bazan NG, Birkle DL, Tang W, Reddy TS (1986) The accumulation of free arachidonic acid, diacylglycerols, prostaglandins, and lipoxygenase reaction products in the brain during experimental epilepsy. Adv Neurol 44:879-902.

Bazan NG, Rodriguez de Turco EB, Allan G (1995) Mediators of injury in neurotrauma: intracellular signal transduction and gene expression. J Neurotrauma 12:791-814.

Bito H, Mori M, Sakanaka C, Takano T, Honda Z, Gotoh Y, Nishida E, Shimizu T (1994) Functional coupling of SSTR4, a major hippocampal somatostatin receptor, to adenylate cyclase inhibition, arachidonate release and activation of the mitogen-activated protein kinase cascade. J Biol Chem 269:12722-12730.

Brewer GJ, Torricelli JR, Evege EK, Price PJ (1993) Optimized survival of hippocampal neurons in B27-supplemented Neurobasal, a new serum-free medium combination. J Neurosci Res 35:567-576.
Chabala LD, Bakry N, Covarrubias M (1993) Low molecular weight poly(A) + mRNA species encode factors that modulate gating of a non-Shaker A-type K+ channel. J Gen Physiol 102:713-728.

Colbert CM, Pan E (1999) Arachidonic acid reciprocally alters the availability of transient and sustained dendritic $\mathrm{K}(+)$ channels in hippocampal CA1 pyramidal neurons. J Neurosci 19:8163-8171.

Dixon JE, Shi W, Wang HS, McDonald C, Yu H, Wymore RS, Cohen IS, McKinnon D (1996) Role of the Kv4.3 K+ channel in ventricular muscle. A molecular correlate for the transient outward current . Circ Res [Erratum (1997) 80:147] 79:659-668.

Drapeau C, Pellerin L, Wolfe LS, Avoli M (1990) Long-term changes of synaptic transmission induced by arachidonic acid in the CA1 subfield of the rat hippocampus. Neurosci Lett 115:286-292.

Dumuis A, Sebben M, Haynes L, Pin JP, Bockaert J (1988) NMDA receptors activate the arachidonic acid cascade system in striatal neurons. Nature 336:68-70.

Dumuis A, Pin JP, Oomagari K, Sebben M, Bockaert J (1990) Arachidonic acid released from striatal neurons by joint stimulation of ionotropic and metabotropic quisqualate receptors. Nature 347:182-184.

Gubitosi-Klug RA, Yu SP, Choi DW, Gross RW (1995) Concomitant acceleration of the activation and inactivation kinetics of the human delayed rectifier $\mathrm{K}+$ channel (Kv1.1) by $\mathrm{Ca}(2+)$-independent phospholipase A2. J Biol Chem 270:2885-2888.

Guo W, Li H, London B, Nerbonne JM (2000) Functional consequences of elimination of I(to, f) and I(to, s): early afterdepolarizations, atrioventricular block, and ventricular arrhythmias in mice lacking Kv1.4 and expressing a dominant-negative Kv4 alpha subunit. Circ Res 87:73-79.

Hoffman DA, Johnston D (1999) Neuromodulation of dendritic action potentials. J Neurophysiol 81:408-411.

Hoffman DA, Magee JC, Colbert CM, Johnston D (1997) K+ channel regulation of signal propagation in dendrites of hippocampal pyramidal neurons. Nature 387:869-875.

Honore E, Attali B, Lesage F, Barhanin J, Lazdunski M (1992) Receptor-mediated regulation of IsK, a very slowly activating, voltagedependent $\mathrm{K}+$ channel in Xenopus oocytes. Biochem Biophys Res Commun 184:1135-1141.

Jerng HH, Covarrubias M (1997) K+ channel inactivation mediated by the concerted action of the cytoplasmic $\mathrm{N}$ - and $\mathrm{C}$-terminal domains. Biophys J 72:163-174.

Jerng HH, Shahidullah M, Covarrubias M (1999) Inactivation gating of Kv4 potassium channels: molecular interactions involving the inner vestibule of the pore. J Gen Physiol 113:641-660.

Johnston D, Hoffman DA, Magee JC, Poolos NP, Watanabe S, Colbert CM, Migliore M (2000) Dendritic potassium channels in hippocampal pyramidal neurons. J Physiol (Lond) 525:75-81.

Keros S, McBain CJ (1997) Arachidonic acid inhibits transient potassium currents and broadens action potentials during electrographic seizures in hippocampal pyramidal and inhibitory interneurons. J Neurosci 17:3476-3487.

Kim D, Duff RA (1990) Regulation of $\mathrm{K}^{+}$channels in cardiac myocytes by free fatty acids. Circ Res 67:1040-1046.

London B, Wang DW, Hill JA, Bennett PB (1998) The transient outward current in mice lacking the potassium channel gene Kv1.4. J Physiol (Lond) 509:171-182.

Madden KS, Kim WT, Cornell-Bell A (1996) Glutamate, arachidonic acid, and calcium regulation in cultured hippocampal astrocytes: involvement in ischemia? Adv Neurol 71:53-59.

Magee J, Hoffman D, Colbert C, Johnston D (1998) Electrical and calcium signaling in dendrites of hippocampal pyramidal neurons. Annu Rev Physiol 60:327-346.

Maletic-Savatic M, Lenn NJ, Trimmer JS (1995) Differential spatiotemporal expression of $\mathrm{K}+$ channel polypeptides in rat hippocampal neurons developing in situ and in vitro. J Neurosci 15:3840-3851.

Meves H (1994) Modulation of ion channels by arachidonic acid. Prog Neurobiol 43:175-186.

Miller B, Sarantis M, Traynelis SF, Attwell D (1992) Potentiation of NMDA receptor currents by arachidonic acid. Nature 355:722-725.

Miller TM, Johnson Jr EM (1996) Metabolic and genetic analyses of apoptosis in potassium/serum-deprived rat cerebellar granule cells. J Neurosci 16:7487-7495.

Needleman P, Turk J, Jakschik BA, Morrison AR, Lefkowith JB (1986) Arachidonic acid metabolism. Annu Rev Biochem 55:69-102.

Pongs O, Leicher T, Berger M, Roeper J, Bahring R, Wray D, Giese KP, Silva AJ, Storm JF (1999) Functional and molecular aspects of voltage-gated $\mathrm{K}+$ channel beta subunits. Ann NY Acad Sci 868:344-355.

Rettig J, Heinemann SH, Wunder F, Lorra C, Parcej DN, Dolly JO, Pongs O (1994) Inactivation properties of voltage-gated K+ channels altered by presence of beta-subunit. Nature 369:289-294.

Rudy B, Hoger JH, Lester HA, Davidson N (1988) At least two mRNA species contribute to the properties of rat brain A-type potassium channels expressed in Xenopus oocytes. Neuron 1:649-658.

Scott VE, Rettig J, Parcej DN, Keen JN, Findlay JB, Pongs O, Dolly JO (1994) Primary structure of a beta subunit of alpha-dendrotoxin- 
sensitive $\mathrm{K}+$ channels from bovine brain. Proc Natl Acad Sci USA 91:1637-1641.

Serodio P, Rudy B (1998) Differential expression of Kv4 K+ channel subunits mediating subthreshold transient $\mathrm{K}+$ (A-type) currents in rat brain. J Neurophysiol 79:1081-1091.

Serodio P, Kentros C, Rudy B (1994) Identification of molecular components of A-type channels activating at subthreshold potentials. J Neurophysiol 72:1516-1529.

Serodio P, Vega- Saenz de Miera E, Rudy B (1996) Cloning of a novel component of A-type $\mathrm{K}+$ channels operating at subthreshold potentials with unique expression in heart and brain. $\mathrm{J}$ Neurophysiol 75:2174-2179.

Sheng M, Tsaur ML, Jan YN, Jan LY (1992) Subcellular segregation of two A-type $\mathrm{K}+$ channel proteins in rat central neurons. Neuron 9:271-284.

Shi G, Nakahira K, Hammond S, Rhodes KJ, Schechter LE, Trimmer JS
(1996) Beta subunits promote $\mathrm{K}+$ channel surface expression through effects early in biosynthesis. Neuron 16:843-852.

Siesjo BK, Agardh CD, Bengtsson F, Smith ML (1989) Arachidonic acid metabolism in seizures. Ann NY Acad Sci 559:323-339.

Villarroel A, Schwarz TL (1996) Inhibition of the Kv4 (Shal) family of transient $\mathrm{K}+$ currents by arachidonic acid. J Neurosci $16: 2522-2532$.

Xu H, Dixon JE, Barry DM, Trimmer JS, Merlie JP, McKinnon D, Nerbonne JM (1996) Developmental analysis reveals mismatches in the expression of $\mathrm{K}+$ channel alpha subunits and voltage-gated $\mathrm{K}+$ channel currents in rat ventricular myocytes. J Gen Physiol 108:405-419.

Xu H, Li H, Nerbonne JM (1999) Elimination of the transient outward current and action potential prolongation in mouse atrial myocytes expressing a dominant negative Kv4 alpha subunit. J Physiol (Lond) 519:11-21. 